Research Paper

\title{
Circular RNA Signature in Hepatocellular Carcinoma
}

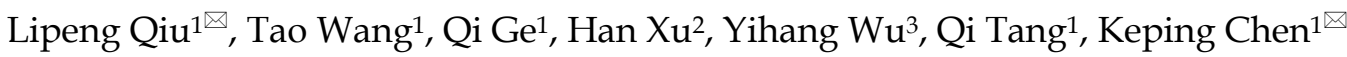 \\ 1. Institute of Life Sciences, Jiangsu University, Zhenjiang 212013, Jiangsu, China \\ 2. School of Pharmacy, Jiangsu University, Zhenjiang, Jiangsu Province, 212013, China. \\ 3. Department of Pharmacy, College of Life Sciences, China Jiliang University, Hangzhou 310018, Zhejiang, China \\ $\bowtie$ Corresponding authors: Lipeng Qiu, E-mail: tulip_lipeng@163.com and Keping Chen, E-mail: kpchen@ujs.edu.cn. \\ (c) Ivyspring International Publisher. This is an open access article distributed under the terms of the Creative Commons Attribution (CC BY-NC) license \\ (https://creativecommons.org/licenses/by-nc/4.0/). See http://ivyspring.com/terms for full terms and conditions.
}

Received: 2018.11.05; Accepted: 2019.05.06; Published: 2019.06.09

\begin{abstract}
Hepatocellular carcinoma (HCC) is one of the most common cancers in the world. Circular RNAs (circRNAs) are a new class of endogenous functional non-coding RNAs (ncRNAs), and have been demonstrated to play important roles in the development of HCC. This study aimed to explore the significance of circRNAs in HCC progression. HCC-associated circRNA expression profiles GSE94508 and GSE97332 were downloaded from the Gene Expression Omnibus database (GEO), and 87 differentially expressed circRNAs (DECs) between HCC tissues and paired non-cancer tissues were identified, including 76 up-regulated and 11 down-regulated circRNAs. Gene ontolog (GO) and pathway analyses of the host genes of these DECs suggested that these host genes were enriched in cell adhesion, cytosol, and protein binding, and were associated with tight junction and Wnt signaling pathways. CircRNA-miRNA interaction prediction identified 20 miRNAs that predispose to interact with DECs. Among these, four essential miRNAs, hsa-miR-7-5p, hsa-miR-145-5p, hsa-miR-203a-3p, and hsa-miR-192-5p, were reported to play pivotal roles in HCC progression by targeting multiple genes. Pathway analysis suggested that putative target genes of these essential miRNAs were involved in HCC-associated signaling pathways, such as Wnt, TGF- $\beta$, and Ras; whereas protein-protein network (PPI) analysis demonstrated that some validated target genes of these miRNAs, such as PIK3CA, AKT1, MYC, JUN, SMAD4, and SRC, were hub target genes as they have more counts of interacting protein. In the meantime, the deregulation of some DECs was validated in HCC cell line HepG2 compared with normal liver cell line LO2 by quantitative real-time polymerase chain reaction (qRT-PCR) and the Sanger sequencing. This study identified a set of DECs in HCC, and provided a comprehensive understanding of the roles of these DECs in $\mathrm{HCC}$ progression.
\end{abstract}

Key words: circular RNA, miRNA sponge, hepatocellular carcinoma, bioinformatic analysis

\section{Introduction}

Hepatocellular carcinoma (HCC), the fifth common cancer in the world, ranks the third in cancer-related death [1]. Currently, the diagnosis of HCC is still poor, and therapeutic options are limited. Many HCC patients are not diagnosed until they are in advanced stages when curative therapies are too late to be applied; while for those patients who are eligible for surgical resection, liver transplantation or local ablation, although their life qualities could be improved by these therapies, their overall survival rates are still low. Therefore, novel biomarkers and therapeutic approaches are urgently needed to improve the overall survival of HCC patients.

Circular RNAs (circRNAs) are a new class of abundant endogenous functional non-coding RNAs (ncRNAs), and have been demonstrated to play important roles in different types of cancer, including gastric cancer, lung cancer, and HCC [2-4]. They form covalently closed continuous loop structures with neither polarity nor polyadenylated tails, which make them more stable and abundant than their canonical linear transcripts from the same genes in cells $[5,6]$. Except for tissues, circRNAs can also be found in different body fluids, such as saliva, blood, and urine, 
suggesting that they may serve as non-invasive circulating biomarkers for cancer diagnosis [7]. Studies showed that circRNAs may regulate gene expression at transcriptional, post-transcriptional, and translational levels. Specifically, circular exonic circRNAs, which predominantly exist in cytoplasm, harbor microRNA (miRNA) response elements (MREs), and may function as miRNA sponges by competitively binding to specific miRNAs to reduce their expression and function, resulting in enhanced expression of target genes of these miRNAs; while circular intronic RNAs or exon-intron circRNAs are mainly accumulate in the nucleus, and may regulate gene transcription and post-transcription [5, 8-10]. These suggest that circRNAs may participate in various biological processes, such as cell proliferation, migration, invasion and metastasis, as well as apoptosis, and thus contribute to cancer progression.

Most recently, some circRNAs have been reported to be deregulated in HCC tissues, and the deregulation of these circRNAs may not only associate with clinicopathological features of HCC patients, but also impact on HCC progression by targeting specific miRNAs and proteins. Several circRNAs, such as circZKSCAN1, hsa_circ_0004018, and hsa_circ_0005075, have been identified as promising biomarkers for HCC diagnosis; whereas circMTO1 and circ-ITCH may serve as potential prognostic biomarkers for poor survival of HCC patients [11-15]. Furthermore, some circRNAs have been reported to interfere with HCC progression by modulating the proliferation, apoptosis, migration and invasion via targeting different miRNAs and proteins. For example, circMTO1 inhibits the proliferation of HCC cells by sponging miR-9 and increasing the expression of miR-9 target gene p21, a cell cycle inhibitory protein; hsa_circ_100338 promotes the migration and invasion of HCC cells by targeting miR-141; while ciRS-7 facilitates the proliferation and invasion of HCC cells by competitively binding to hsa-miR-7-5p (miR-7) and promoting the expression of multiple miR-7 target genes, including CCNE1, PIK3CD, and EGFR [14, 16-19]. These results indicate that circRNAs may play critical roles in HCC progression.

In the current study, we downloaded the HCC-associated circRNA expression profiles GSE945 08 and GSE97332 from the Gene Expression Omnibus database (GEO), analyzed the differentially expressed circRNAs (DECs) in both profiles between paired HCC and non-tumor liver tissues, and investigated their possible target genes and pathways involved in HCC using bioinformatic approaches, to provided possible functions and underlying mechanisms of these DECs in HCC tumorigenesis and progression.

\section{Materials and methods}

\section{Microarray analysis of gene expression}

Two circRNA expression profiles, GSE94508 and GSE97332, were downloaded from the GEO (www.ncbi.nlm.nih.gov/geo/), both of which were completed on the Agilent-069978 Arraystar Human CircRNA microarray V1 GPL19978 platform [20]. The GSE94508 dataset contained 5 pairs of HCC and paracancerous liver tissues, and the GSE97332 dataset contained 7 pairs of HCC and matched non-tumor liver tissues.

\section{Identification of DECs}

GEO2R [21] was used to analysis the raw data TXT files downloaded from GEO. The absolute value of $\log$ fold change $>1.0$ and $p$ value $<0.05$ were used as cut-off criteria. CircRNAs with statistical significance between HCC and non-tumor tissues in GSE94508 and GSE97332 were screened, separately. Then, circRNAs up-regulated or down-regulated in both profiles were selected and identified.

\section{Functional enrichment analysis}

Gene ontolog (GO) annotation, including biological process, cellular component, and molecular function, was analyzed using the Database for Annotation, Visualization, and Integrated Discovery (DAVID; https://david. ncifcrf.gov) [22-24]. Kyoto Encyclopedia of Genes and Genomes (KEGG) and Panther (http.//www.pantherdb.org/) pathway analyses were used to classify genomic and gene functional information [25-26]. The significant enrichment results were obtained with $p$ values $<0.05$.

\section{Prediction of circRNA-miRNA and miRNA-mRNA interactions}

Online tools miRDB (http.//www.mirdb.org/) and circinteractome (https.//circinteractome.nia.nih. gov/) were used to predict the possible interactions between circRNAs and miRNAs [27-29]; miRDB and TargetScan (http.//www.targetscan.org/mamm_31/ ) were applied for predicting target genes of the four essential miRNAs; while mirTarBase (http./ / mirtarba se.mbc.nctu.edu.tw/php/index.php) was used to obtain experimentally strongly supported targets genes (by Reporter assay, Western blot, or qPCR) of these miRNAs [30-32].

\section{Construction of protein-protein interaction (PPI) network}

Experimentally supported target genes of the four essential miRNAs were put into the Search Tool for the Retrieval of Interacting Genes database (STRING; https.//string-db.org/), and an interaction network chart with a combined score $>0.4$ was saved 
and exported [33]. Subsequently, top 50 genes with more counts of interacting protein were selected, and the PPI network was visualized using Cytoscape software (version 3.6.1; http.//cytoscape.org/) [34]. The values of gene interactions predicted by STRING were also imported into Cytoscape to identify hub genes among potential targets.

\section{Cell culture}

HCC cell line HepG2 was cultured in Dulbecco's Modified Eagle Medium (DMEM, Gibco, USA), and human normal liver cell line L02 was cultured in 1640 in a humidified $37^{\circ} \mathrm{C}$ incubator with $5 \% \mathrm{CO}_{2}$. Both media were supplemented with 10\% FBS (Gibco. USA), $2 \mathrm{mmol} / \mathrm{L}$ L-glutamine and $100 \mathrm{U} / \mathrm{mL}$ penicillin/100 $\mu \mathrm{g} / \mathrm{mL}$ streptomycin (Life Technologies, CA, USA).

\section{RNA extraction and quantitative real-time polymerase chain reaction ( $q R T-P C R$ )}

Total RNAs from HepG2 and L02 cells were extracted using TRIzol reagent (Life Technologies, CA, USA) according to the manufacture's protocol. RNA quality and quantity was meatured using Synergy H4 Hybrid Multi-Mode Microplate Reader (BioTek Instruments Inc, Winooski, VT, USA). Total RNAs were reversely transcribe using HiScript Q RT SuperMix for qPCR with gDNA wiper (Vazyme Biotech, Nanjing, China), and qPCR assays were performed in triplicate using AceQ qPCR SYBR Green Master Mix kit (Vazyme Biotech, Nanjing, China) on the ABI QuantStudio 3 real-time PCR system (ThermoFisher Scientific, USA). The divergent primers used for detecting circRNAs were synthesized from Shanghai Generay Biotech (Shanghai, China), and $\beta$-actin was used as an internal control. The sequences of primers for qPCR were listed in Table 1, and the specificity of PCR products was evaluated by dissociation curve analysis and the Sanger sequencing (Sango Biotech, Shanghai, China).

Table 1. Circular RNA (circRNA) primers used for qPCR

\begin{tabular}{|c|c|c|}
\hline Gene & Forward primer $\left(5^{\prime}-3^{\prime}\right)$ & Reverse primer $\left(5^{\prime}-3^{\prime}\right)$ \\
\hline has_circ_0001806 & $\begin{array}{l}\text { CCATCCCATCAGTTCAT } \\
\text { ССТ }\end{array}$ & $\begin{array}{l}\text { TTCACCTCCAAAGAGC } \\
\text { ATCC }\end{array}$ \\
\hline has_circ_0003528 & $\begin{array}{l}\text { GTAACCAGCAGCCTGG } \\
\text { ACTC }\end{array}$ & $\begin{array}{l}\text { GCAACTTGCTGACCAG } \\
\text { AACA }\end{array}$ \\
\hline has & $\begin{array}{l}\text { TTACGGGAGCAGATGA } \\
\text { TGAA }\end{array}$ & $\begin{array}{l}\text { CCAAGAAGGAAGATGG } \\
\text { GCTA }\end{array}$ \\
\hline has & $\begin{array}{l}\text { CAGGTTCTGGACGTCA } \\
\text { AAGG }\end{array}$ & $\begin{array}{l}\text { TCACCTCAGCCATGTGT } \\
\text { СTC }\end{array}$ \\
\hline has_c & $\begin{array}{l}\text { TTGTTCCTCATCGCAGC } \\
\text { AGT }\end{array}$ & $\begin{array}{l}\text { ATAGAGGCGCACGTCA } \\
\text { AACT }\end{array}$ \\
\hline has_circ_0065214 & $\begin{array}{l}\text { TCATGTCTGTGGGACTC } \\
\text { TGC }\end{array}$ & $\begin{array}{l}\text { GGGCGAGTAATCCTTC } \\
\text { ACAG }\end{array}$ \\
\hline has_circ_0007762 & $\begin{array}{l}\text { CATTCAGATGGCACCTT } \\
\text { GAC }\end{array}$ & $\begin{array}{l}\text { GTGCCCACATAGAGCC } \\
\text { ACTT }\end{array}$ \\
\hline$\beta$-actin & $\begin{array}{l}\text { AGAAAATCTGGCACCA } \\
\text { CACC }\end{array}$ & $\begin{array}{l}\text { CAGAGGCGTACAGGGA } \\
\text { TAGC }\end{array}$ \\
\hline
\end{tabular}

\section{Statistical analysis}

Statistical analyses were performed using GraphPad Prism 5 (GraphPad Software, CA, USA). All Data were shown as mean \pm SEM. Student's $t$-test was used to compare the differences of circRNA expression between L02 and HepG2 cells, and a $p$ value $<0.05$ was considered statistically significant.

\section{Results}

\section{Selection of DECs}

Two circRNA expression profiles, including GSE94508 and GSE97332, were downloaded from GEO, and GEO2R method was applied to analysis DECs between HCC liver tissues and paired nontumor tissues. Results showed that many circRNAs were differentially expressed in HCC tissues. In GSE94508, 560 circRNAs were identified to be differentially expressed, including 214 up-regulated and 346 down-regulated circRNAs; while in GSE97332, 892 differentially expressed circRNAs were identified, including 453 up-regulated and 439 down-regulated circRNAs. Among them, 87 circRNAs, including 76 up-regulated and 11 downregulated circRNAs, were observed in both circRNA expression profiles (Figure 1A and 1B, and Table 2).
A

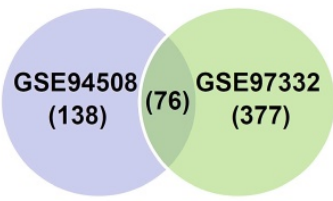

B

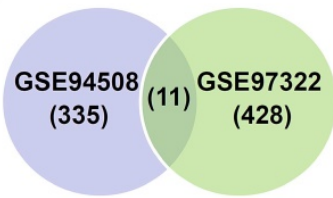

Figure 1. Differentially expressed circular RNAs (DECs) in hepatocellular carcinoma (HCC) compared with paired non-tumor liver tissues. (A-B) Venn diagram represents the overlap of the identified DECs in HCC tissues from the results of microarray GSE94508 (5 pairs) and GSE97332 (7 pairs). (A) Up-regulated circRNAs in HCC tissues. (B) Down-regulated circRNAs in HCC tissues.

\section{GO and pathway analysis of the host genes of DECs}

Since some circRNAs function by regulating their parental genes, GO analysis was carried out to annotate the host genes of the 87 DECs. Biological process analysis showed that these host genes were enriched in cell adhesion, positive/ negative regulation of gene expression, and Golgi organization. Cellular component analysis indicated that these host genes were remarkably enriched in cytosol, membrane and Golgi apparatus. For molecular function, the most significantly enriched GO term was protein binding (Figure 2A). KEGG and Panther pathway analysis demonstrated that tight junction, aldosterone synthesis and secretion, inflammation mediated by chemokine and cytokine signaling pathway, and Wnt signaling pathway may involved in circRNA- 
mediated regulatory network in the pathogenesis of HCC (Figure 2B).

\section{Prediction of circRNA-miRNA interactions}

Increasing evidences demonstrate that circRNAs may competitively bind to miRNAs and increase the expression of the target genes of these miRNAs. For the 87 DECs, prediction by online tools miRDB and circinteractome revealed 343 and 1085 circRNA-
miRNA interactions, respectively. Furthermore, 20 consensus miRNAs from both prediction tools were identified, and DECs potentially bind to these miRNAs were presented in Table 3. Results showed that one specific circRNA may bind to more miRNAs, while different circRNAs could interact with one specific miRNA, indicating that circRNAs may impact on HCC progression by modulating various miRNAs.

Table 2. Differentially expressed circRNAs (DECs) in hepatocellular carcinoma (HCC)

\begin{tabular}{|c|c|c|c|c|}
\hline No. & CircRNA ID & Chromosome location & Gene symbol & Accession number \\
\hline \multicolumn{5}{|c|}{ Up-regulated circRNAs } \\
\hline 1 & hsa_circ_0000291 & chr11:35163017-35163328+ & CD44 & NM_001202557 \\
\hline 2 & hsa_circ_0000511 & chr14:20811282-20811431- & RPPH1 & NR_002312 \\
\hline 3 & hsa_circ_0000554 & chr14:74551677-74551959+ & LIN52 & NM_001024674 \\
\hline 4 & hsa_circ_0000644 & chr15:81195758-81195954- & KIAA1199 & NM_018689 \\
\hline 5 & hsa_circ_0000673 & chr16:11940357-11940700- & RSL1D1 & NM_015659 \\
\hline 6 & hsa_circ_0000747 & chr17:27209637-27210251+ & FLOT2 & NM_004475 \\
\hline 7 & hsa_circ_0000981 & chr2:20240809-20240905- & LAPTM4A & NM_014713 \\
\hline 8 & hsa_circ_0001228 & chr22:37868480-37870715- & MFNG & NM_002405 \\
\hline 9 & hsa_circ_0001279 & chr3:33109721-33110462- & GLB1 & NM_001079811 \\
\hline 10 & hsa_circ_0001338 & chr3:128824688-128825122- & RAB43 & NM_001204883 \\
\hline 11 & hsa_circ_0001489 & chr5:59770958-59771235+ & PDE4D & NM_001165899 \\
\hline 12 & hsa_circ_0001806 & chr8:68018139-68028357+ & CSPP1 & NM_024790 \\
\hline 13 & hsa_circ_0001828 & chr8:142139086-142139265+ & DENND3 & NM_014957 \\
\hline 14 & hsa_circ_0001834 & chr9:2017333-2017502+ & SMARCA2 & NM_003070 \\
\hline 15 & hsa_circ_0001901 & chr9:138773785-138774005- & CAMSAP1 & NM_015447 \\
\hline 16 & hsa_circ_0001917 & chrX:41519691-41530783- & CASK & NM_001126055 \\
\hline 17 & hsa_circ_0001955 & chr15:64495280-64508912- & CSNK1G1 & NM_022048 \\
\hline 18 & hsa_circ_0002191 & chr9:97535283-97563284+ & C9orf3 & NM_001193331 \\
\hline 19 & hsa_circ_0002702 & chr9:35546426-35548532+ & RUSC2 & NM_001135999 \\
\hline 20 & hsa_circ_0003528 & chr5:134032815-134044578+ & SEC24A & NM_021982 \\
\hline 21 & hsa_circ_0003645 & chr16:19656207-19663412+ & C16orf62 & NM_020314 \\
\hline 22 & hsa_circ_0003892 & chr19:11230767-11238761+ & LDLR & NM_000527 \\
\hline 23 & hsa_circ_0003923 & chr2:238933982-238940895+ & UBE2F & NM_080678 \\
\hline 24 & hsa_circ_0003945 & chr9:33953282-33956144- & UBAP2 & NM_018449 \\
\hline 25 & hsa_circ_0003958 & chr7:27668989-27672064- & HIBADH & NM_152740 \\
\hline 26 & hsa_circ_0004004 & chr5:172359438-172362313+ & ERGIC1 & NM_001031711 \\
\hline 27 & hsa_circ_0004976 & chr2:25990451-25994409- & ASXL2 & NM_018263 \\
\hline 28 & hsa_circ_0005397 & chr17:30500849-30503232+ & RHOT1 & NM_001033568 \\
\hline 29 & hsa_circ_0005785 & chr12:110819556-110834257- & ANAPC7 & NM_016238 \\
\hline 30 & hsa_circ_0006517 & chr3:188202379-188242575+ & LPP & NM_005578 \\
\hline 31 & hsa_circ_0006789 & chrX:118544152-118544325+ & SLC25A43 & NM_145305 \\
\hline 32 & hsa_circ_0007196 & chr3:50144199-50147121+ & RBM5 & NM_005778 \\
\hline 33 & hsa_circ_0008274 & chr13:96485180-96489456- & UGGT2 & NM_020121 \\
\hline 34 & hsa_circ_0008310 & chr17:7849045-7849304+ & CNTROB & NM_001037144 \\
\hline 35 & hsa_circ_0008563 & chr1:21599191-21599404- & ECE1 & NM_001113347 \\
\hline 36 & hsa_circ_0008583 & chr3:196817782-196846401- & DLG1 & NM_004087 \\
\hline 37 & hsa_circ_0009910 & chr1:12049221-12052747+ & MFN2 & NM_014874 \\
\hline 38 & hsa_circ_0012107 & chr1:44290402-44303983+ & ST3GAL3 & NM_174963 \\
\hline 39 & hsa_circ_0013048 & chr1:82302569-82372915+ & LPHN2 & NM_012302 \\
\hline 40 & hsa_circ_0014879 & chr1:160206924-160231148- & DCAF8 & NR_028103 \\
\hline 41 & hsa_circ_0016404 & chr1:212977661-212977993+ & TATDN3 & NM_001146171 \\
\hline 42 & hsa_circ_0018004 & chr10:27024168-27024508+ & PDSS1 & NM_014317 \\
\hline 43 & hsa_circ_0026143 & chr12:49722709-49723237+ & TROAP & NM_005480 \\
\hline 44 & hsa_circ_0027478 & chr12:69109406-69125499+ & NUP107 & NM_020401 \\
\hline 45 & hsa_circ_0028196 & chr12:110826316-110834257- & ANAPC7 & NM_016238 \\
\hline 46 & hsa_circ_0029325 & chr12:125270902-125284788- & SCARB1 & NM_005505 \\
\hline 47 & hsa_circ_0031132 & chr14:21464367-21464870+ & METTL17 & NM_022734 \\
\hline 48 & hsa_circ_0032704 & chr14:76173360-76187046+ & TTLL5 & NM_015072 \\
\hline 49 & hsa_circ_0033408 & chr14:102842986-102931626+ & TECPR2 & NM_014844 \\
\hline 50 & hsa_circ_0036005 & chr15:67004005-67008836+ & SMAD6 & NR_027654 \\
\hline 51 & hsa_circ_0038718 & chr16:27351506-27353580+ & IL4R & NM_000418 \\
\hline 52 & hsa_circ_0039053 & chr16:30510394-30510810+ & ITGAL & NM_002209 \\
\hline 53 & hsa_circ_0043438 & chr17:37583953-37584043- & MED1 & NM_004774 \\
\hline
\end{tabular}




\begin{tabular}{|c|c|c|c|c|}
\hline No. & CircRNA ID & Chromosome location & Gene symbol & Accession number \\
\hline 54 & hsa_circ_0045006 & chr17:59152280-59161925+ & BCAS3 & NM_001099432 \\
\hline 55 & hsa_circ_0045862 & chr17:76082583-76083174+ & TNRC6C & NM_001142640 \\
\hline 56 & hsa_circ_0048937 & chr19:6934997-6937659+ & EMR1 & NM_001974 \\
\hline 57 & hsa_circ_0049997 & chr19:17626981-17628198+ & PGLS & NM_012088 \\
\hline 58 & hsa_circ_0051220 & chr19:41884185-41884424+ & TMEM91 & NM_001098821 \\
\hline 59 & hsa_circ_0051732 & chr19:48660270-48660397- & LIG1 & NM_000234 \\
\hline 60 & hsa_circ_0060055 & chr20:33866724-33872064- & EIF6 & NM_181468 \\
\hline 61 & hsa_circ_0062682 & chr22:26936754-26937684- & TPST2 & NM_001008566 \\
\hline 62 & hsa_circ_0064288 & chr3:11348416-11350535+ & ATG7 & NM_006395 \\
\hline 63 & hsa_circ_0065214 & chr3:47466974-47476627- & SCAP & NM_012235 \\
\hline 64 & hsa_circ_0067934 & chr3:170013698-170015181+ & PRKCI & NM_002740 \\
\hline 65 & hsa_circ_0072088 & chr5:32379220-32388780- & ZFR & NM_016107 \\
\hline 66 & hsa_circ_0073271 & chr5:88044886-88047860- & MEF2C & NM_002397 \\
\hline 67 & hsa_circ_0074903 & chr5:168110970-168112932- & SLIT3 & NM_003062 \\
\hline 68 & hsa_circ_0078738 & chr6:170033042-170058454- & WDR27 & NM_182552 \\
\hline 69 & hsa_circ_0082182 & chr7:128317617-128323309+ & FAM71F2 & NM_001128926 \\
\hline 70 & hsa_circ_0082564 & chr7:137569740-137570248- & CREB3L2 & NM_194071 \\
\hline 71 & hsa_circ_0083766 & chr8:27382878-27394372+ & EPHX2 & NM_001979 \\
\hline 72 & hsa_circ_0091331 & chrX:109310574-109352374+ & TMEM164 & NM_032227 \\
\hline 73 & hsa_circ_0092283 & chr22:36681395-36681695- & MYH9 & NM_002473 \\
\hline 74 & hsa_circ_0092298 & chr5:178287348-178287568+ & ZNF354B & NM_058230 \\
\hline 75 & hsa_circ_0092310 & chr6:43467278-43467478+ & TJAP1 & NM_001146018 \\
\hline 76 & hsa_circ_0092327 & chr19:17972481-17972901+ & RPL18A & NM_000980 \\
\hline \multicolumn{5}{|c|}{ Down-regulated circRNAs } \\
\hline 1 & hsa_circ_0003859 & chr19:41122794-41125398+ & LTBP4 & NM_001042544 \\
\hline 2 & hsa_circ_0004913 & chr17:62248459-62265775- & TEX2 & NM_018469 \\
\hline 3 & hsa_circ_0005428 & chr1:47761436-47770668- & STIL & NM_001048166 \\
\hline 4 & hsa_circ_0007762 & chr6:147581750-147599340+ & STXBP5 & NM_001127715 \\
\hline 5 & hsa_circ_0008160 & chr21:38439561-38441924- & PIGP & NR_028352 \\
\hline 6 & hsa_circ_0036044 & chr15:68434283-68457142+ & PIAS1 & NM_016166 \\
\hline 7 & hsa_circ_0038929 & chr16:29810948-29811369+ & KIF22 & NM_007317 \\
\hline 8 & hsa_circ_0043302 & chr17:36353600-36353765- & LOC440434 & NR_036750 \\
\hline 9 & hsa_circ_0051637 & chr19:47285639-47285806- & SLC1A5 & NM_005628 \\
\hline 10 & hsa_circ_0056548 & chr2:135878388-135881816+ & RAB3GAP1 & NM_001172435 \\
\hline 11 & hsa_circ_0078279 & chr6:151226785-151293194+ & MTHFD1L & NM_001242767 \\
\hline
\end{tabular}

Table 3. Consensus microRNAs (miRNAs) and DECs potentially interact with them.

\begin{tabular}{|c|c|}
\hline MiRNAs & DECs predicted to interact with \\
\hline hsa-miR-1248 & $\begin{array}{l}\text { hsa_circ_0032704, hsa_circ_0000673, hsa_circ_0039053, hsa_circ_0016404, hsa_circ_0028196, hsa_circ_0003958, } \\
\text { hsa_circ_0004976, hsa_circ_0008583, hsa_circ_0003528, hsa_circ_0001806, hsa_circ_0078279, hsa_circ_0005428 }\end{array}$ \\
\hline hsa-miR-1236-3p & $\begin{array}{l}\text { hsa_circ_0032704, hsa_circ_0016404, hsa_circ_0027478, hsa_circ_0065214, hsa_circ_0001806, hsa_circ_0065214, } \\
\text { hsa_circ_0002191, hsa_circ_0014879, hsa_circ_0007762, hsa_circ_0003859 }\end{array}$ \\
\hline hsa-miR-330-5p & $\begin{array}{l}\text { hsa_circ_0001338, hsa_circ_0000291, hsa_circ_0005397, hsa_circ_0003892, hsa_circ_0007196, hsa_circ_0082182, } \\
\text { hsa_circ_0002702, hsa_circ_0043302, hsa_circ_0026143, hsa_circ_0001955 }\end{array}$ \\
\hline hsa-miR-615-5p & $\begin{array}{l}\text { hsa_circ_0008563, hsa_circ_0045006, hsa_circ_0092283, hsa_circ_0009910, hsa_circ_0002702, hsa_circ_0001228, } \\
\text { hsa_circ_0006789, hsa_circ_0000511, hsa_circ_0003859 }\end{array}$ \\
\hline hsa-miR-1299 & $\begin{array}{l}\text { hsa_circ_0039053, hsa_circ_0002702, hsa_circ_0003645, hsa_circ_0002191, hsa_circ_0056548, hsa_circ_0007762, } \\
\text { hsa_circ_0001806, hsa_circ_0001955, hsa_circ_0083766 }\end{array}$ \\
\hline hsa-miR-486-3p & $\begin{array}{l}\text { hsa_circ_0002702, hsa_circ_0003859, hsa_circ_0001806, hsa_circ_0000554, hsa_circ_0000291, hsa_circ_0001806, } \\
\text { hsa_circ_0033408, hsa_circ_0092310 }\end{array}$ \\
\hline hsa-miR-370-3p & $\begin{array}{l}\text { hsa_circ_0004976, hsa_circ_0003528, hsa_circ_0074903, hsa_circ_0003945, hsa_circ_0001338, hsa_circ_0026143, } \\
\text { hsa_circ_0062682 }\end{array}$ \\
\hline hsa-miR-589-5p & hsa_circ_0005428, hsa_circ_0000291, hsa_circ_0003892, hsa_circ_0008583, hsa_circ_0002191, hsa_circ_0078279 \\
\hline hsa-miR-145-5p & hsa_circ_0001955, hsa_circ_0028196, hsa_circ_0083766, hsa_circ_0001489, hsa_circ_0027478, hsa_circ_0005428 \\
\hline hsa-miR-1286 & hsa_circ_0000747, hsa_circ_0026143, hsa_circ_0038718, hsa_circ_0051732, hsa_circ_0003958, hsa_circ_0002702 \\
\hline hsa-miR-7-5p & hsa_circ_0028196, hsa_circ_0065214, hsa_circ_0005785, hsa_circ_0003892, hsa_circ_0001489, hsa_circ_0092327 \\
\hline hsa-miR-637 & hsa_circ_0016404, hsa_circ_0039053, hsa_circ_0026143, hsa_circ_0004913, hsa_circ_0003892, hsa_circ_0005397 \\
\hline hsa-miR-377-3p & hsa_circ_0036044, hsa_circ_0003859, hsa_circ_0072088, hsa_circ_0008274, hsa_circ_0003645 \\
\hline hsa-miR-203a-3p & hsa_circ_0039053, hsa_circ_0013048, hsa_circ_0032704, hsa_circ_0078279 \\
\hline hsa-miR-1296-5p & hsa_circ_0001955, hsa_circ_0001489, hsa_circ_0001279, hsa_circ_0082564 \\
\hline hsa-miR-192-5p & hsa_circ_0003528, hsa_circ_0078738, hsa_circ_0007196 \\
\hline hsa-miR-1256 & hsa_circ_0033408, hsa_circ_0002191, hsa_circ_0001955 \\
\hline hsa-miR-1289 & hsa_circ_0049997, hsa_circ_0004976, hsa_circ_0008160 \\
\hline hsa-miR-215-5p & hsa_circ_0003528, hsa_circ_0078738 \\
\hline hsa-miR-1261 & hsa_circ_0072088, hsa_circ_0009910 \\
\hline
\end{tabular}




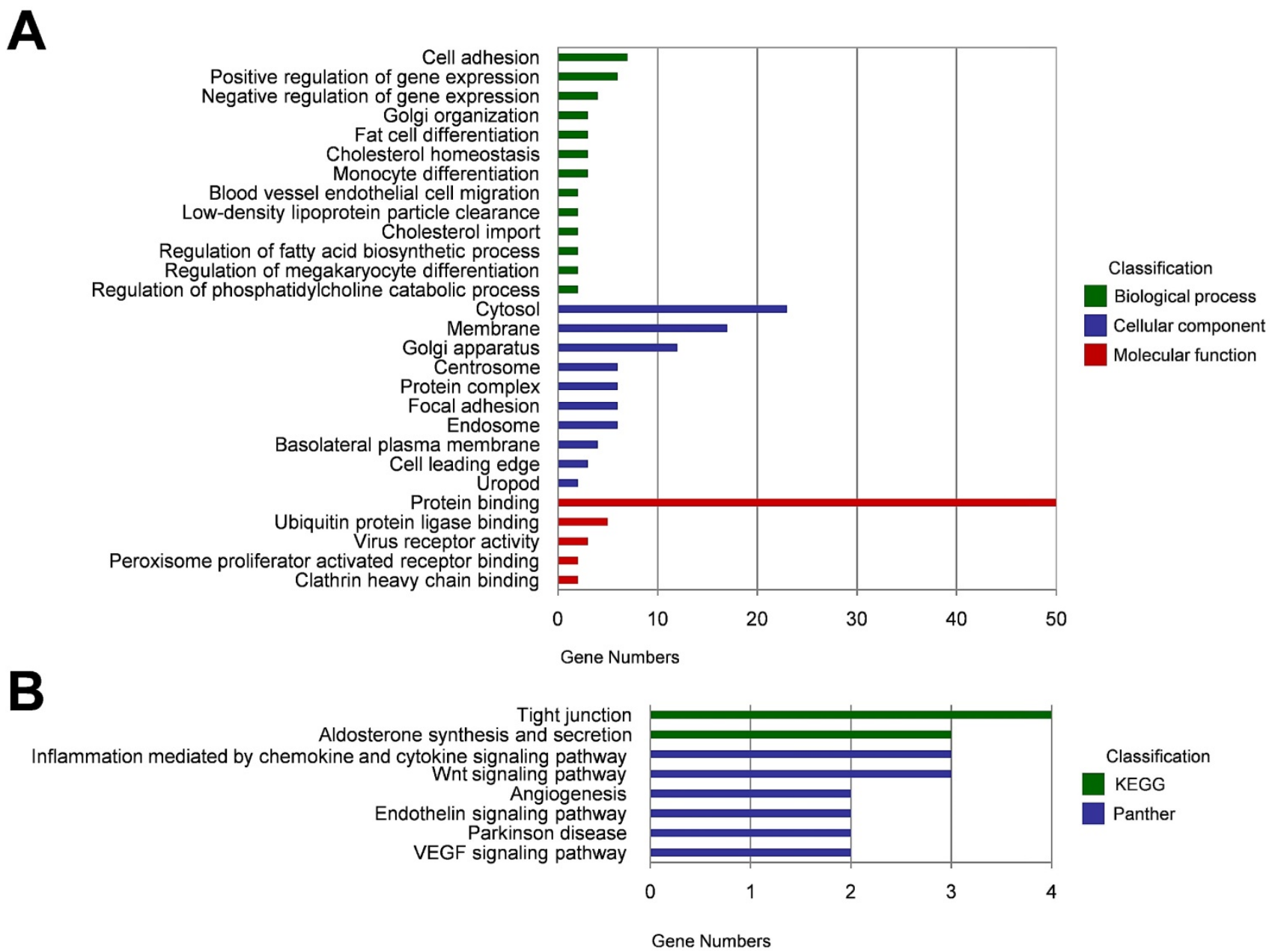

Figure 2. Functional annotation of the host genes of DECs. (A) Gene ontolog (GO) enrichment analyses of the host genes of DECs, including biological process, cellular component, and molecular function. (B) KEGG and Panther pathway enrichment analyses of the host genes of DECs.

\section{Target genes of some essential miRNAs and their function analysis}

Among the 20 consensus miRNAs, four essential miRNAs, including miR-7, hsa-miR-145-5p (miR-145), hsa-miR-203a-3p (miR-203a), and hsa-miR-192-5p (miR-192), were reported to be down-regulated in $\mathrm{HCC}$, and play pivotal roles in HCC progression by targeting multiple protein-coding genes. Using online tools miRDB and TargetScan, 1480 target genes of these four miRNAs were predicted, and then presented in Venn diagram (Figure 3). Two common target genes (SLC4A4 and ZDHHC9) were found for miR-7, miR-145 and miR-203a; two target genes (CPEB4, DYRK1A) were found for miR-145, miR-203a, and miR-192; and one target gene (MECP2) for miR-7, miR-203a and miR-192; while none of these genes was predicted to be targeted by all the four miRNAs. KEGG and Panther pathway analyses revealed that these target genes were involved in many HCC-associated signaling pathways, such as Wnt, TGF- $\beta$, and Ras, suggesting their possible roles in HCC pathogenesis (Figure 4).
miR-145 target genes miR-203a target genes

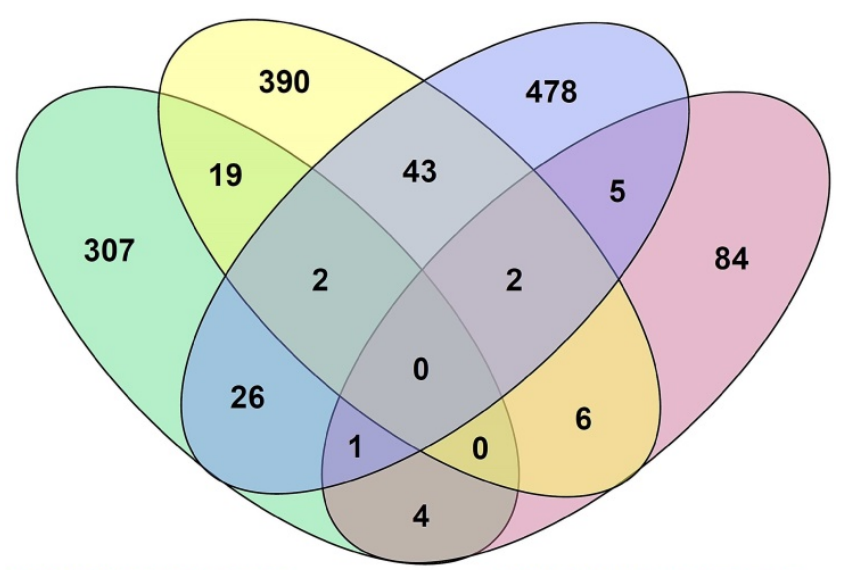

miR-7 target genes

miR-192 target genes

Figure 3. Venn diagram of target genes of the four essential microRNAs (miRNAs), including hsa-miR-7-5p (miR-7), hsa-miR -145-5p (miR-145), hsa-miR-203a-3p (miR-203), and hsa-miR-192-5p (miR-192). 1480 target genes of the four miRNAs were predicted using online tools miRDB and TargetScan. 
A

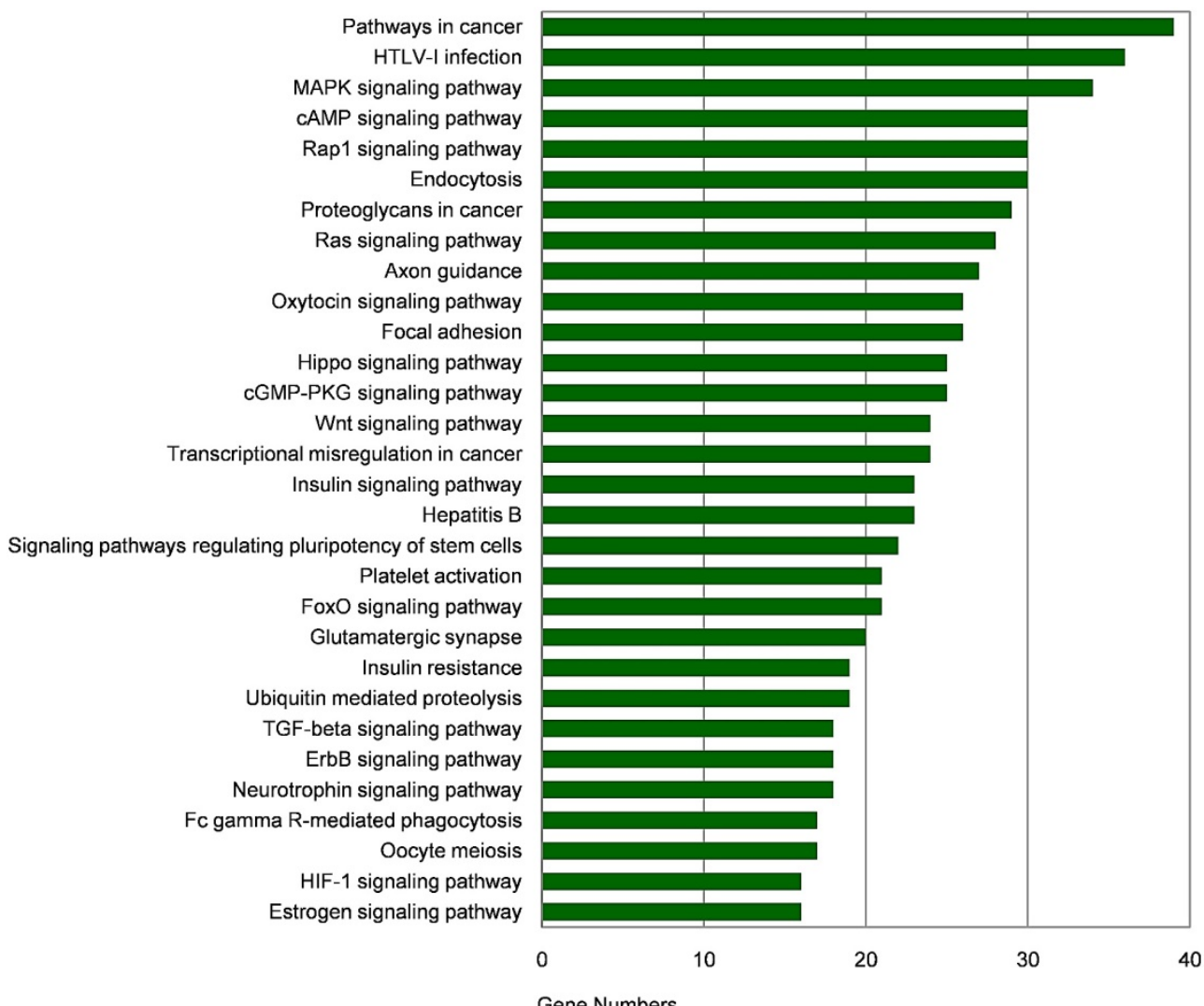

B

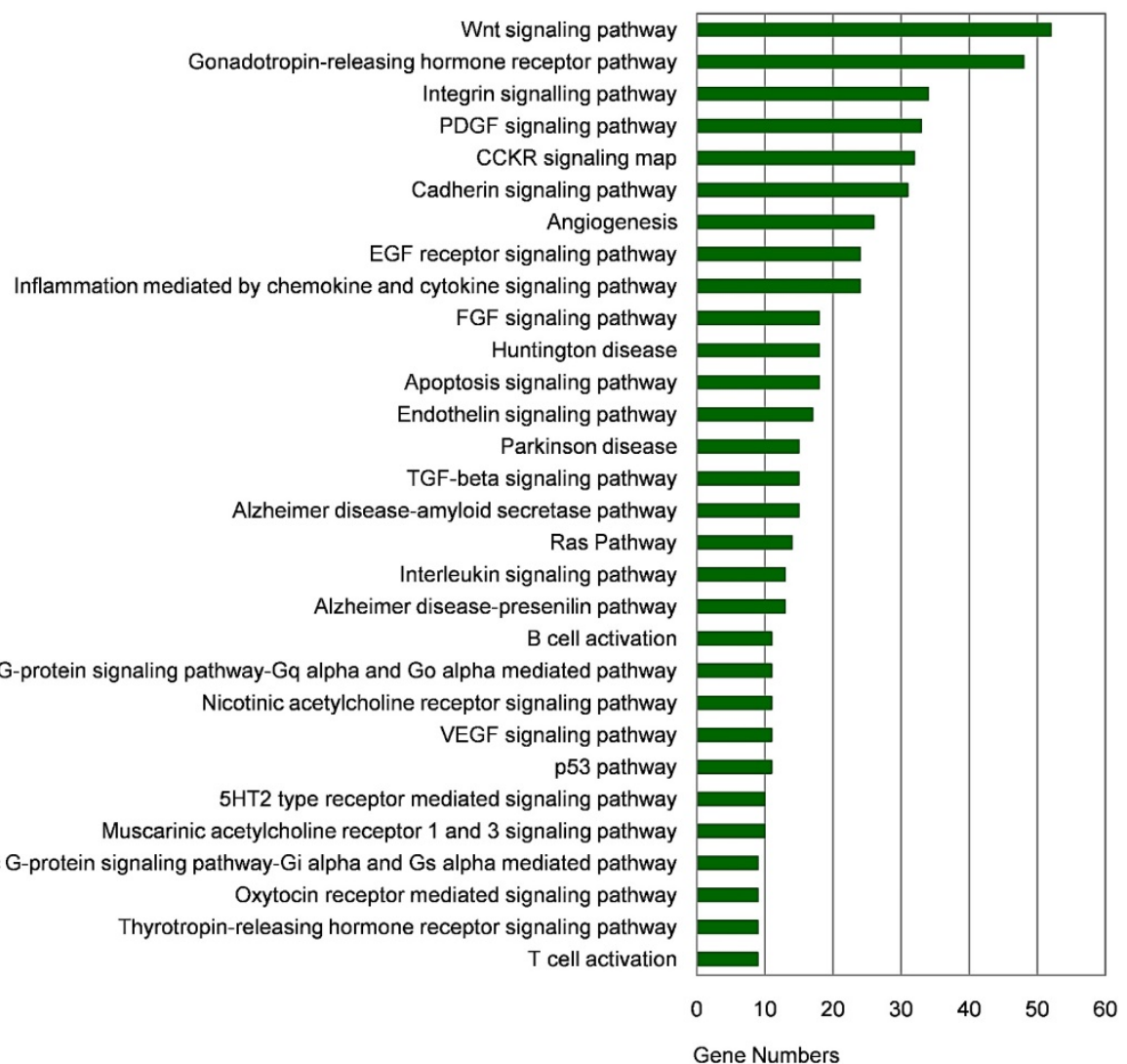

Figure 4. Pathway analysis of target genes of the four essential miRNAs. (A-B) KEGG pathway (A) and Panther pathway (B) functional analyses of these target genes. 


\section{Construction of essential miRNAs-centered regulatory network}

To analysis the significance of target genes of the four essential miRNAs, their experimentally strongly supported targets genes were obtained from mirTarBase. Among these, some genes, such as ABCC1 and CUL5, have been demonstrated to be targeted by more than one essential miRNAs, suggesting a complex regulatory network involving the four essential miRNAs and their target genes. Furthermore, protein-protein interaction (PPI) was analyzed using online tool STRING, and the PPI network of the top 50 genes which have more counts of interacting protein was visualized using Cytoscape software (Figure 5). Subsequently, four essential miRNAs and their potential interacting circRNAs were combined into the regulatory network. In this figure, proteins with bigger circles showed more interactions with other proteins than those with smaller circles.

\section{Validation of DECs}

To verify the deregulation of DECs, nine of them were randomly chosen for further validation. The expression of these DECs in L02 and HepG2 cells were detected using qRT-PCR, and the specificity of qPCR products was evaluated by dissociation curve analysis and the Sanger sequencing. Among them, the expression of two circRNAs, including hsa_circ 0001489 and hsa_circ_0039053, was low in both cell lines, and was failed to be detected. Six circRNAs were validated to be greatly up-regulated in HepG2 cells compared with L02 cells, including hsa_circ_ 0001806, hsa_circ_0003528, hsa_circ_0008583, hsa_circ _0009910, hsa_circ_0032704, and hsa_circ_0065214 (Figure 6A-6F); while hsa_circ_0007762 was found to be greatly down-regulated in HepG2 cells compared with L02 cells (Figure 6G). The expression pattern of these circRNAs in HCC cell lines was similar to that in HCC specimens from the two GEO HCC datasets.

\section{Discussion}

Emerging evidences indicate that circRNAs are frequently deregulated in HCC tissues. Some deregulated circRNAs may serve as biomarkers for HCC diagnosis and prognosis, while others could regulate HCC progression through diverse mechanisms by functioning as miRNA sponges, RNA-binding protein sponges, or transcriptional regulators [4]. In the current study, we identified 87 DECs from the HCC circRNA expression profiles of GSE94508 and GSE97332 downloaded from GEO database, and analyzed the functions and possible underlying mechanisms of these DECs in HCC tumorigenesis and progression using bioinformatic methods. In the meantime, the deregulation of some DECs was validated in HCC cells by qRT-PCR and the Sanger sequencing.

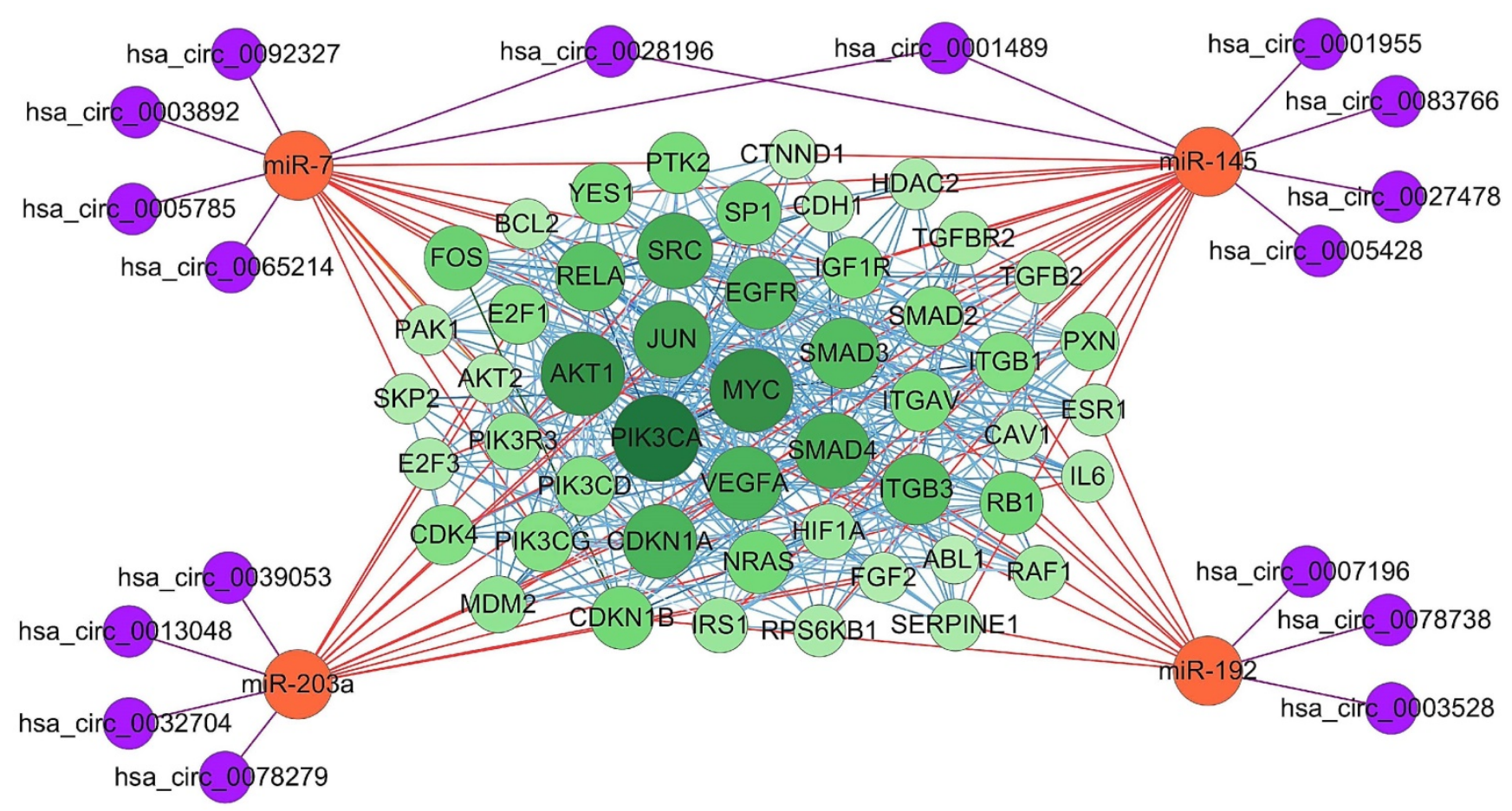

Figure 5. Essential miRNAs-centered circRNA-miRNA-mRNA network. The experimentally supported target genes of the four essential miRNAs were obtained using online tool mirTarBase. MiRNA-mRNA interaction was represented as orange edges, protein-protein interaction was represented as blue edges, and circRNA-miRNA interaction was represented as purple edges. PIK3CA, AKT1, MYC, JUN, SMAD4, and SRC were shown as hub target genes as they have more counts of interacting protein. 


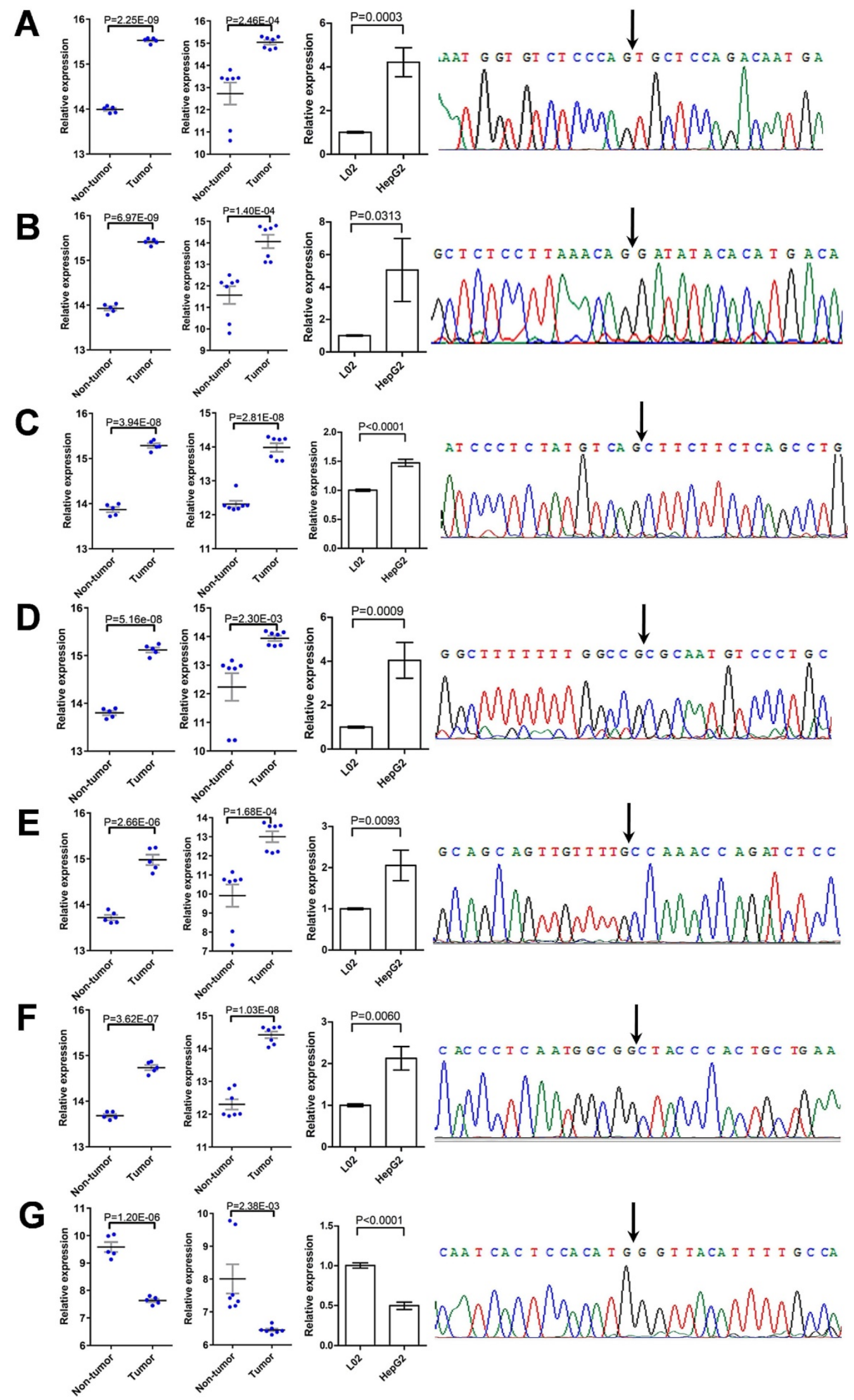

Figure 6. Validation of DECs. (A-G) The deregulation of seven DECs, including hsa_circ_0001806 (A), hsa_circ_0003528 (B), hsa_circ_0008583 (C), hsa_circ_0009910 (D), hsa circ 0032704 (E), hsa circ 0065214 (F), and hsa circ 0007762 (G), was validated by GRT-PCR and the Sanger sequencing. For each DEC, from left to right: comparison of relative expression of the $\mathrm{DE} \bar{C}$ between $\mathrm{HCC}$ tissues and paired non-tumor tissues, as extracted from GSE94508 (five pairs) and GSE97332 (seven pairs), respectively; comparison of relative expression of the DEC between HCC cell line HepG2 and normal liver cell line L02, as determined by qRT-PCR; and the specificity of the divergent PCR products, as verified by the Sanger sequencing of the DEC in back-splice junction. Data were shown as mean \pm SEM, and student's t-test was used to compare the differences of circRNA expression between two groups. 
Among the 87 DECs identified in our study, four up-regulated circRNAs, including hsa_circ_0000 673, hsa_circRNA_0072088, hsa_circ_0009910, and hsa_circ_0067934, have been reported to play important roles in the progression of different cancers, such as HCC, gastric cancer (GC), colorectal cancer (CRC), and esophageal squamous cell carcinoma (ESCC) [35-40]. Two circRNAs, hsa_circ_0000673 and hsa_circRNA_0072088, were up-regulated in HCC and lung cancer, separately, and play tumorpromoting roles by modulating miR-767-3p/SET axis and miR-4302/znf121/MYC axis, respectively [35, 37]. However, they were down-regulated, and may act as tumor suppressors in other cancers. Hsa_circ 0000673 was reported to inhibit tumor suppressor gene RUNX3 by targeting miR-532-5p, leading to inhibition of GC progression; while hsa_circRNA_007 2088 was found to promote FOXO4 by targeting miR-532-3p, resulting in suppression of CRC development $[36,38]$. Other two circRNAs, hsa_circ 0009910 and hsa_circ_0067934, were demonstrated to be up-regulated in osteosarcoma and ESCC, separately, and play oncogenic roles in these cancers. Hsa_circ_0009910 was capable of sponging miR-499a and enhancing the expression of miR-499 target gene IL6R, and thus accelerating carcinogenesis of osteosarcoma; while hsa_circ_0067934 could promote the proliferation and migration of ESCC cells, and its expression level in ESCC was associated with tumor differentiation and TNM stages [39, 40]. Interestingly, our study demonstrated the up-regulation of hsa_circ_009910 in HCC, indicating its possible roles in HCC progression. The precise function and mechanisms of this circRNA in HCC is currently under investigation.

Recent research works suggest that some circRNAs may regulate the transcription of their parental genes [41]. In the current study, the possible GO functional terms and signaling pathways of the host genes of these DECs were characterized. Results showed that these host genes were enriched in cell adhesion, cytosol, and protein binding, all of which were associated with HCC progression. Pathway analysis from KEGG and Panther demonstrated that tight junction and Wnt signaling pathway are among the most relevant pathways for HCC. Tight junction signaling contributes to pathogenesis of GC and CRC, plays functional roles in epithelial-to-mesenchymal transition and viral entry, and could be used as potential targets for gastrointestinal and liver disease; while Wnt signaling pathway is well known to impact on hepatocarcinogenesis and HCC progression [42-45]. These results suggested that these DECs may participate in HCC progression by regulating their parental genes.
Many miRNAs have been demonstrated to regulate HCC progression by modulating the expression of one or more HCC-associated genes [46, 47]. However, the functions of miRNAs can be interfered by their possible interaction with circRNAs and lncRNAs. One of the most interesting examples is the interaction between ciRS-7 and miR-7. CiRS-7 is a special and unique natural circRNA which harbors more than 70 binding sites for miR-7. By binding to miR-7, ciRS-7 facilitates the expression of miR-7 target genes, such as CCNE1, PIK3CD, and EGFR, and thus blocks the inhibitory effect of miR-7 on HCC [17-19]. Similarly, circMTO1 and circHIPK3 were found to modulate HCC progression by competitively binding to miR-9 and miR-124-3p, respectively, and thus increasing the expression of multiple oncogenic genes, such as p21, Norch1, AQP3 [14, 48]. In the current study, the interactions between DECs and miRNAs were predicted. Among all the DECs, hsa_circ_0005428 contains the most number of interactions and could potentially bind to 16 different miRNAs, whereas hsa_circ_0001834 and hsa_circ_ 000511 have only one potential miRNA binding site. Among the top 20 miRNAs which predispose to interact with DECs, each one contains at least two potential DECs binding sites. Specially, hsa-miR-1248 has as many as 12 DECs binding sites. Given the importance of miRNAs in HCC progression, it is possible that DECs could impact on HCC progression by targeting specific miRNAs to increasing the expression of their target genes.

Among the top 20 miRNAs, four essential miRNAs, including miR-7, miR-145, miR-203, and miR-192, were reported to be down-regulated in HCC, and could inhibit HCC progression by modulating the expression of multiple target genes; while their modulation on target genes may be blocked by their interaction with non-coding RNAs. For example, interaction between miR-7 and ciRS-7 or lncRNA KCNQ1OT1 led to reduced expression of miR-7 and increased expression of miR-7 target genes CCNE1, PIK3CD, EGFR, and ABCC1, resulting in promotion of HCC progression [18, 19, 49]; whereas interaction between miR-145 and lincRNA-ROR or pseudogene OCT4-pg4 led to reduction of this miRNA and increase of miR-145 target genes RAD18, ZEB2, and OCT4, resulting in aggravation of HCC [50-52]. MiR-203 could interact with three lncRNAs, including CRNDE, UCA1 and HULC, and the interaction of which greatly elevated the expression of miR-203 target genes BCAT1, SNAI2, and ADAM9, and thus facilitating HCC progression [53-55]; while miR-192, however, could be blocked by IncRNA HOTTIP, and reduced expression of this miRNA led to exacerbated expression of its target gene GLS1, 
resulting in enhanced progression of HCC [56]. Regarding to their pivotal roles in HCC progression, these four essential miRNAs were selected for further analysis. With their predicted and validated target genes, pathway and protein-protein interaction network analyses were carried out. KEGG and Panther pathway analyses suggested that the predicted target genes of these miRNAs were involved in various pathways, such as Wnt, TGF- $\beta$, oxytoxin, and Ras, all of which are closely related to hepatogenesis and HCC progression. A four essential miRNAs-centered circRNA-miRNA-mRNA network was established by combination of the potential interacting DECs of the four miRNAs with the PPI network based on the top 50 experimentally supported target genes of these miRNAs. Results suggested that some target genes of these miRNAs, such as PIK3CA, AKT1, MYC, JUN, SMAD4, and SRC, may impact on cell function by interacting with different molecules in cells and interfering with various signaling pathways, while specific circRNAs may control the expression of these genes by indirectly targeting essential miRNAs. As expected, we found that the expression of two circRNAs, including hsa_circ_0032704 and hsa_circ_0065214, was greatly increased in HCC tissues and cell lines, suggesting that these two circRNAs may function in HCC progression by targeting HCC suppressor genes miR-203a and miR-7, respectively, leading to enhanced expression of oncogenic proteins and promotion of HCC progression. Currently, their functions and molecular mechanisms, including possible interactions with miRNAs, are under investigation.

In conclusion, this study revealed aberrantly expressed circRNAs in HCC, and discussed their possible roles in HCC progression. Further investigations are needed to fully elucidate the functions and underlying mechanisms of these deregulated circRNAs and their regulatory networks in tumorigenesis and HCC progression.

\section{Abbreviations}

HCC: Hepatocellular carcinoma; circRNAs: circular RNAs; ncRNAs: non-coding RNAs; GEO: the Gene Expression Omnibus database; DECs: differentially expressed circRNAs; miRNA: microRNA; MREs: miRNA response elements; GO: Gene ontolog; PPI: protein-protein network; qRT-PCR: quantitative real-time polymerase chain reaction; miR-7: hsa-miR7-5p; DAVID: the Database for Annotation, Visualization, and Integrated Discovery; KEGG: Kyoto Encyclopedia of Genes and Genomes; STRING: the Search Tool for the Retrieval of Interacting Genes database; miR-145: hsa-miR-145-5p; miR-203a: hsa-miR-203a3p; miR-192: hsa-miR-192-5p; GC: gastric cancer;
CRC: colorectal cancer; ESCC: esophageal squamous cell carcinoma.

\section{Acknowledgements}

This work was supported by the National Natural Science Foundation of China (81402840, 31402016, and 31572467); the National Key R\&D Program of China (2018YFA0108400), the Natural Science Foundation of Jiangsu Province, China (BK20130495); the Training Project of Young Backbone Teachers of Jiangsu University, and Jiangsu University Senior Professional Science Foundation (11JDG120).

\section{Competing Interests}

The authors have declared that no competing interest exists.

\section{References}

1. Farazi PA, DePinho RA. Hepatocellular carcinoma pathogenesis. from genes to environment. Nat Rev Cancer. 2006; 6: 674-687.

2. Zhang J, Liu H, Hou L, et al. Circular RNA_LARP4 inhibits cell proliferation and invasion of gastric cancer by sponging miR-424-5p and regulating LATS1 expression. Mol Cancer. 2017; 16: 151.

3. $\mathrm{Hu} \mathrm{W}, \mathrm{Bi} Z \mathrm{ZY}, \mathrm{Chen} \mathrm{ZL}$, et al. Emerging landscape of circular RNAs in lung cancer. Cancer Lett. 2018; 427: 18-27.

4. Qiu LP, $\mathrm{Wu} \mathrm{YH}, \mathrm{Yu} \mathrm{XF}$, et al. The Emerging Role of Circular RNAs in Hepatocellular Carcinoma. J Cancer. 2018; 9: 1548-1559.

5. Jeck WR, Sorrentino JA, Wang K, et al. Circular RNAs are abundant, conserved, and associated with ALU repeats. RNA. 2013; 19.141-157.

6. Suzuki H, Tsukahara T. A view of pre-mRNA splicing from RNase R resistant RNAs. Int J Mol Sci. 2014; 15: 9331-9342.

7. Qu S, Yang X, Li X, et al. Circular RNA. A new star of noncoding RNAs. Cancer Lett. 2015; 365: 141-148.

8. Salzman J, Chen RE, Olsen MN, et al. Cell-type specific features of circular RNA expression. PLoS Genet. 2013; 9: e1003777.

9. Memczak S, Jens M, Elefsinioti A, et al. Circular RNAs are a large class of animal RNAs with regulatory potency. Nature. 2013; 495: 333-338.

10. $\mathrm{Yu}$ B, Shan G. Functions of long noncoding RNAs in the nucleus. Nucleus. 2016; 7: 155-166.

11. Yao Z, Luo J, Hu K, et al. ZKSCAN1 gene and its related circular RNA (circZKSCAN1) both inhibit hepatocellular carcinoma cell growth, migration, and invasion but through different signaling pathways. Mol Oncol. 2017; 11: 422-437.

12. Fu L, Yao $\mathrm{T}$, Chen $\mathrm{Q}$, et al. Screening differential circular RNA expression profiles reveals hsa_circ_0004018 is associated with hepatocellular carcinoma. Oncotarget. 2017; 8: 58405-58416.

13. Shang X, Li G, Liu H, et al. Comprehensive Circular RNA Profiling Reveals That hsa circ 0005075, a New Circular RNA Biomarker, Is Involved in Hepatocellular Crcinoma Development. Medicine (Baltimore). 2016; 95: e3811.

14. Han D, Li J, Wang H, et al. Circular RNA circMTO1 acts as the sponge of microRNA-9 to suppress hepatocellular carcinoma progression. Hepatology. 2017; 66: 1151-1164.

15. Guo W, Zhang J, Zhang D, et al. Polymorphisms and expression pattern of circular RNA circ-ITCH contributes to the carcinogenesis of hepatocellular carcinoma. Oncotarget. 2017; 8: 48169-48177.

16. Huang $\mathrm{XY}$, Huang ZL, $\mathrm{Xu}$ YH, et al. Comprehensive circular RNA profiling reveals the regulatory role of the circRNA-100338/miR-141-3p pathway in hepatitis B-related hepatocellular carcinoma. Sci Rep. 2017; 7: 5428.

17. Xu L, Zhang M, Zheng X, et al. The circular RNA ciRS-7 (Cdr1as) acts as a risk factor of hepatic microvascular invasion in hepatocellular carcinoma. J Cancer Res Clin Oncol. 2017; 143: 17-27.

18. Yu L, Gong X, Sun L, et al. The Circular RNA Cdr1as Act as an Oncogene in Hepatocellular Carcinoma through Targeting miR-7 Expression. PLoS One. 2016; 11: e0158347.

19. Yang $\mathrm{X}$, Xiong $\mathrm{O}, \mathrm{Wu} \mathrm{Y}$, et al. Quantitative Proteomics Reveals the Regulatory Networks of Circular RNA CDR1as in Hepatocellular Carcinoma Cells. J Proteome Res. 2017; 16: 3891-3902.

20. Barrett T, Suzek TO, Troup DB, et al. NCBI GEO. mining millions of expression profiles--database and tools. Nucleic Acids Res. 2005; 33: D562-566.

21. Barrett T, Troup DB, Wilhite SE, et al. NCBI GEO. mining tens of millions of expression profiles--database and tools update. Nucleic Acids Res. 2007; 35: D760-765. 
22. Huang DW, Sherman BT, Lempicki RA. Systematic and integrative analysis of large gene lists using DAVID Bioinformatics Resources. Nature Protoc. 2009; 4: 44-57.

23. Huang DW, Sherman BT, Lempicki RA. Bioinformatics enrichment tools. paths toward the comprehensive functional analysis of large gene lists. Nucleic Acids Res. 2009; 37: 1-13.

24. Gene Ontology Consortium. GO: The Gene Ontology (GO) project in 2006. . Nucleic Acids Res. 2006; 34: D322-D326.

25. Mi H, Dong Q, Muruganujan A, et al. PANTHER version 7: improved phylogenetic trees, orthologs and collaboration with the Gene Ontology Consortium. Nucleic Acids Res. 2010; 38: D204-D210.

26. Kanehisa M, Goto S. KEGG: kyoto encyclopedia of genes and genomes. Nucleic Acids Res. 2000; 28: 27-30.

27. Wong N, Wang X. miRDB: an online resource for microRNA target prediction and functional annotations. Nucleic Acids Res. 2015; 43: D146-152.

28. Wang $X$. Improving microRNA target prediction by modeling with unambiguously identified microRNA-target pairs from CLIP-ligation studies. Bioinformatics. 2016; 32: 1316-1322.

29. Dudekula DB, Panda AC, Grammatikakis I, et al. CircInteractome: A web tool for exploring circular RNAs and their interacting proteins and microRNAs. RNA Biology. 2016; 13: 34-42.

30. Lewis BP, Shih IH, Jones-Rhoades MW, et al. Prediction of mammalian microRNA targets. Cell. 2003; 115:787-798.

31. Lewis BP, Burge CB, Bartel DP. Conserved seed pairing, often flanked by adenosines, indicates that thousands of human genes are microRNA targets. Cell. 2005; 120: 15-20.

32. Chou CH, Shrestha S, Yang CD, et al. miRTarBase update 2018: a resource for experimentally validated microRNA-target interactions. Nucleic Acids Res. 2018; 46: D296-D302.

33. Jensen L, Kuhn M, Stark M, et al. STRING 8--a global view on proteins and their functional interactions in 630 organisms. Nucleic Acids Res 2009; 37: D412-D416.

34. Shannon P, Markiel A, Ozier O, et al. Cytoscape: a software environment for integrated models of biomolecular interaction networks. Genome Res. 2003; 13: 2498-2504.

35. Jiang W, Wen D, Gong L, et al. Circular RNA hsa_circ_0000673 promotes hepatocellular carcinoma malignance by decreasing miR-767-3p targeting SET. Biochem Biophys Res Commun. 2018; 500: 211-216.

36. Chang P, Wang F, Li Y. Hsa_circ_0000673 is down-regulated in gastric cancer and inhibits the proliferation and invasion of tumor cells by targetting miR-532-5p. Biosci Rep 2018; 38: BSR20180538.

37. Liu W, Ma W, Yuan Y, et al. Circular RNA hsa_circRNA_103809 promotes lung cancer progression via facilitating ZNF121-dependent MYC expression by sequestering miR-4302. Biochem Biophys Res Commun. 2018; 500: 846-851.

38. Bian L, Zhi X, Ma L, et al. Hsa circRNA 103809 regulated the cell proliferation and migration in colorectal cancer via miR-532-3p / FOXO4 axis. Biochem Biophys Res Commun. 2018; 505: 346-352.

39. Deng N, Li L, Gao J, et al. Hsa_circ 0009910 promotes carcinogenesis by promoting the expression of miR-449a target IL6R in osteosarcoma. Biochem Biophys Res Commun. 2018; 495: 189-196.

40. Xia W, Qiu M, Chen R, et al. Circular RNA has_circ_0067934 is upregulated in esophageal squamous cell carcinoma and promoted proliferation. Sci Rep. 2016; 6: 35576.

41. Li Z, Huang C, Bao C, et al. Exon-intron circular RNAs regulate transcription in the nucleus. Nat Struct Mol Biol. 2015; 22: 256-264.

42. Zeisel MB, Dhawan P, Baumert TF. Tight junction proteins in gastrointestinal and liver disease. Gut 2018; [Epub ahead of print].

43. Sekhar V, Pollicino T, Diaz G, et al. Infection with hepatitis C virus depends on TACSTD2, a regulator of claudin-1 and occludin highly downregulated in hepatocellular carcinoma. PLoS Pathog. 2018; 14: e1006916.

44. Liu LJ, Xie SX, Chen YT, et al. Aberrant regulation of Wnt signaling in hepatocellular carcinoma. World J Gastroenterol. 2016; 22: 7486-7499.

45. Blagotinsek K, Rozman D. Targeting Signalling Pathways in Hepatocellular Carcinoma. Curr Pharm Des. 2017; 23: 170-175.

46. $\mathrm{Xu} \mathrm{X}, \mathrm{Tao} \mathrm{Y}$, Shan $\mathrm{L}$, et al. The Role of MicroRNAs in Hepatocellular Carcinoma. J Cancer. 2018; 9: 3557-3569.

47. Xie KL, Zhang YG, Liu J, et al. MicroRNAs associated with HBV infection and HBV-related HCC. Theranostics. 2014; 4: 1176-1192.

48. Chen G, Shi Y, Liu M, et al. circHIPK3 regulates cell proliferation and migration by sponging miR-124 and regulating AQP3 expression in hepatocellular carcinoma. Cell Death Dis. 2018; 9: 175.

49. $\mathrm{Hu} \mathrm{H}$, Yang $\mathrm{L}$, Li L, et al. Long non-coding RNA KCNQ1OT1 modulates oxaliplatin resistance in hepatocellular carcinoma through miR-7-5p/ ABCC1 axis. Biochem Biophys Res Commun. 2018; 503: 2400-2406.

50. Chen $\mathrm{Y}$, Shen $\mathrm{Z}$, Zhi $\mathrm{Y}$, et al. Long non-coding RNA ROR promotes radioresistance in hepatocelluar carcinoma cells by acting as a ceRNA for microRNA-145 to regulate RAD18 expression. Arch Biochem Biophys. 2018; 645: 117-125.

51. Li C, Lu L, Feng B, et al. The lincRNA-ROR/miR-145 axis promotes invasion and metastasis in hepatocellular carcinoma via induction of epithelial-mesenchymal transition by targeting ZEB2. Sci Rep. 2017; 7: 4637.

52. Wang L, Guo ZY, Zhang R, et al. Pseudogene OCT4-pg4 functions as a natural micro RNA sponge to regulate OCT4 expression by competing for miR-145 in hepatocellular carcinoma. Carcinogenesis. 2013; 34: 1773-1781.
53. Ji D, Jiang C, Zhang L, et al. LncRNA CRNDE promotes hepatocellular carcinoma cell proliferation, invasion, and migration through regulating miR-203/ BCAT1 axis. J Cell Physiol. 2019; 234: 6548-6560.

54. Xiao JN, Yan TH, Yu RM, et al. Long non-coding RNA UCA1 regulates the expression of Snail2 by miR-203 to promote hepatocellular carcinoma progression. J Cancer Res Clin Oncol. 2017; 143: 981-990.

55. Wan D, Shen S, Fu S, et al. miR-203 suppresses the proliferation and metastasis of hepatocellular carcinoma by targeting oncogene ADAM9 and oncogenic long non-coding RNA HULC. Anticancer Agents Med Chem. 2016; 16: 414-423.

56. Ge Y, Yan X, Jin Y, et al. fMiRNA-192 and miRNA-204 Directly Suppress lncRNA HOTTIP and Interrupt GLS1-Mediated Glutaminolysis in Hepatocellular Carcinoma. PLoS Genet. 2015; 11: e1005726. 